\title{
Les interactions sociales comme frein à l'expression de la sensibilité environnementale des jeunes
}

Jocelyn Lachance

\section{(2) OpenEdition}

Journals

Édition électronique

URL : https://journals.openedition.org/ere/8229

DOI : $10.4000 /$ ere.8229

ISSN : 2561-2271

Éditeur

Centr'ERE

\section{Référence électronique}

Jocelyn Lachance, «Les interactions sociales comme frein à l'expression de la sensibilité

environnementale des jeunes », Éducation relative à l'environnement [En ligne], Volume 16-2 | 2021, mis en ligne le 14 octobre 2021, consulté le 08 février 2022. URL : http://journals.openedition.org/ere/8229 ; DOI : https://doi.org/10.4000/ere.8229

Ce document a été généré automatiquement le 8 février 2022.

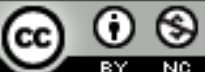

La revue Éducation relative à l'environnement est mise à disposition selon les termes de la Licence Creative Commons Attribution - Pas d'Utilisation Commerciale 4.0 International. 


\title{
Les interactions sociales comme frein à l'expression de la sensibilité environnementale des jeunes
}

\author{
Jocelyn Lachance
}

1 Les réseaux sociaux se situent depuis plusieurs années au cœur de la sociabilité juvénile (Boyd, 2014). Non seulement sont-ils devenus des lieux d'échanges entre jeunes, ils jouent désormais un rôle central dans la réception, le partage et le traitement d'informations (Cordier, 2015). Le sujet des enjeux environnementaux n'échappe pas à cette tendance, et des études montrent à cet effet l'importance que prennent ces réseaux sociaux pour la diffusion d'informations sur la situation environnementale, mais aussi pour l'échange de pratiques écoresponsables (Boulianne, 2015). Les réseaux sociaux apparaissent alors comme des moyens de renforcer la sensibilité environnementale des jeunes, que Louise Chawla (1998) définit comme une prédisposition pour le goût d'apprendre à propos de l'environnement, à se sentir concerné par cet environnement et à agir pour le conserver sur la base d'expériences significatives (Pruneau et Gravel, 2004). Or, comme nous le verrons, si les réseaux sociaux jouent des rôles diversifiés pour faciliter l'échange d'information, voire pour favoriser la rencontre entre jeunes sensibles aux causes environnementales, ils ne facilitent pas toujours l'expression des convictions personnelles à ce sujet. En effet, afficher son désir d'engagement comporte aux yeux des jeunes se déclarant engagés dans le domaine de l'environnement, une part de prise de risque que certains affrontent et que d'autres redoutent, ce qui semblent constituer des freins à l'expression de l'engagement en faveur de l'environnement, à une époque où les réseaux sociaux se présentent souvent comme des opportunités en ce domaine (Conroy et coll., 2012; Bouliane, 2019). En analysant le discours de nos répondants au sujet de l'affichage de leurs convictions à ce sujet sur les réseaux sociaux, nous verrons que l'affirmation de soi en ligne ne relève pas toujours de l'évidence, ce qui questionne l'importance que prend à leurs yeux le jugement des autres. Nos analyses nous amènent alors à questionner l'importance de prendre en compte dans le champ de l'éducation 
relative à l'environnement l'écart entre l'existence de convictions chez les jeunes et leur capacité d'exprimer publiquement ces mêmes convictions.

\section{Engagement, expérimentation et prise de risque chez les jeunes}

2 Geoffroy Pleyers définit l'engagement comme « un processus d'expérimentation créatif par lequel sont mises en pratique les valeurs d'un "autre monde" au sein même des associations, ou au cours de la vie quotidienne » $(2014$, p. 3). S'appuyant sur l'analyse de nombreux entretiens réalisés avec des jeunes engagés dans le mouvement altermondialiste, il montre comment les formes d'expression de l'engagement se diversifient jusqu'à s'incarner parfois dans des gestes quotidiens, en dehors de toutes implications dans des actions collectives. Or, l'expérimentation n'est pas qu'un synonyme de diversification, voire d'innovation quant aux manières de s'engager. Elle implique aussi une part d'incertitude pour au moins deux raisons. D'une part, la forme donnée à son engagement n'est jamais parfaitement prévisible. Elle est appelée à se transformer dans le temps, à s'adapter au contexte social et aux opportunités d'agir. D'autre part, l'expérimentation implique toujours une part de doute aux yeux des individus qui la vivent, car il n'est jamais certain que les individus qui en seront les témoins attribuent à celle-ci une valeur positive. En d'autres termes, si l'expérimentation créative de Pleyers implique toujours la chance que celle-ci soit valorisée par autrui, elle suppose aussi le risque qu'elle ne soit pas validée par les autres, voire qu'elle soit dévalorisée. Ainsi, s'il est possible pour un jeune engagé en faveur de la défense de l'environnement de transformer ses modes de consommation ou de proposer à son entourage d'adopter des gestes écoresponsables, il n'est jamais certain que ses décisions personnelles et ses propositions soient entérinées par les autres au cours des interactions.

Dans ce contexte, les jeunes décidant de s'engager en faveur de l'environnement sont amenés à interroger la validité même des formes données à leur engagement et à les revoir au besoin, d'où le «processus créatif » décrit par Pleyers. Le regard que portent leurs parents, leurs amis, leurs enseignants ou encore leur copain ou leur copine sont susceptibles de jouer un rôle important dans la confirmation que ces formes données à leur engagement sont valables ou non. Il ne s'agit alors pas de faire reconnaître par les autres un statut de jeunes engagés, mais bien d'attendre une confirmation de la valeur attribuée à leur action considérée comme étant un symbole de leur engagement. La temporalité de la reconnaissance est alors plus restreinte que lorsque l'on espère être reconnu pour avoir atteint un objectif: il ne suffit plus d'attendre le moment où l'action a engendré ses effets pour chercher une validation. Il importe de trouver cette validation pendant le déroulement même de l'action (Lachance, 2018). Ce qui importe, c'est de se positionner et de se repositionner par rapport aux différents cercles de reconnaissance, et donc par rapport aux différents interlocuteurs dans chaque interaction sociale (Pizzorno, 2009). Ainsi pouvons-nous imaginer les efforts de justification que devront faire des jeunes engagés dans ce domaine lorsqu'ils retrouvent des parents qui doutent de leurs idées ou des moyens pris pour les défendre, ou quelques amis qui leur redemandent s'ils sont certains de la voie qu'ils ont choisie. Leurs tentatives de faire reconnaître les formes données à leur engagement s'expriment alors à chaque fois qu'ils cherchent à faire valider, non pas l'atteinte d'un 
statut de personne engagée, mais bien la valeur et le sens d'une décision, d'une démarche entreprise, d'un choix effectué. L'interaction devient alors le lieu de la validation ou de l'invalidation de l'acte engagé.

4 Le "processus d'expérimentation créatif » décrit par Pleyers s'exprime aussi sur les réseaux sociaux, mais les recherches disponibles peinent à confirmer que ces derniers facilitent l'engagement en faveur de l'environnement chez les jeunes. D'une part, l'engagement politique en ligne est décrit comme étant individualisé et privatisé (Dahlgren, 2012), ce qui fait écho aux tendances observées par les sociologues s'intéressant aux transformations de l'engagement chez les jeunes depuis les dernières décennies (Ion, 1997). Certes, les réseaux sociaux favorisent l'adhésion en ligne à des groupes militants (Valenzuela et coll., 2009), participent de l'augmentation de la conscience environnementale des jeunes (Bell et coll., 2015), facilitent la mobilisation des personnes pour participer à des événements engagés et augmentent les chances d'être invités à participer à des activités engagées (Musick et Wilson, 2008). Mais des travaux récents soulignent aussi qu'une multitude de sujets d'actualité sont discutés par les jeunes sur YouTube, débouchant sur des échanges et des positions concernant des lois ou des débats politiques récents sur le genre, le port du voile, la pornographie, la consommation, le racisme ou l'écologie (Balleys, 2018). Ces échanges sont notamment menés par des célébrités qui mobilisent les TICs pour stimuler des positions politiques, parfois féministes ou écologistes parmi leurs admirateurs (Bennett, 2013). Mais d'autres études rappellent la limite de l'usage des réseaux sociaux comme modalité qui faciliterait l'expression de ses usagers, puisque ces derniers renforceraient les inégalités sociales en augmentant la visibilité des personnalités publiques déjà visibles dans l'espace public (Fuchs, 2014). Dans ce contexte, il reste difficile de confirmer si les réseaux sociaux favorisent l'engagement des jeunes ou si l'engagement des jeunes favorise l'utilisation des réseaux sociaux (Boulianne, 2015). Or, comme nous le verrons dans cet article, les risques perçus par les jeunes qui se déclarent engagés en faveur de l'environnement les retiennent bien souvent d'afficher leurs convictions en ligne. En tant qu'espace « d'expérimentation créatif », les réseaux sociaux apparaissent alors comme des espaces où le regard de l'autre, omniprésent, ralentit bien souvent leur désir d'afficher leurs convictions.

\section{Méthodologie}

5 Dans le cadre de notre projet de recherche ECOTIC $^{1}$ sur le rapport à l'engagement environnemental des jeunes, nous avons contacté plusieurs associations de défense de l'environnement en France afin de rencontrer des jeunes impliqués dans des actions collectives de ce type. Nous avons également lancé des appels sur les réseaux sociaux afin d'interviewer des jeunes se disant engagés en faveur de l'environnement sans pour autant faire partie d'un groupe d'activiste. Cette décision de rassembler des jeunes engagés collectivement et individuellement s'appuie précisément sur les travaux de Geoffroy Pleyers $(2010,2016)$ qui confirme l'individualisation et la privatisation récente de l'engagement environnemental des jeunes. Tous les jeunes avaient cependant en commun de se déclarer comme étant engagé en faveur de la défense de l'environnement. Cela pouvait s'exprimer à travers leur appartenance à un mouvement comme Youth for Climate, leur implication dans une association de protection de l'environnement ou un mode de consommation vécu comme étant une forme 
d'expression de cet engagement. Lors de la prise de contact, il fut expliqué aux jeunes que l'objectif de la recherche était double : d'une part, mieux connaître les définitions et les frontières de leur engagement environnemental, d'autre part, interroger le rôle des réseaux sociaux dans la mise en acte de cet engagement. Au total nous avons recruté 32 jeunes, âgés entre 18 et 25 ans de nationalité française, 14 garçons et 18 filles ; parmi les 32 interviewés, 9 ont déclaré ne pas faire partie d'associations ou de groupes engagés en faveur de l'écologie. Chaque répondant.e fut interviewé.e une fois pour une durée variant entre 42 minutes et $1 \mathrm{~h} 30$. Les interviews se sont déroulés en face à face ou par visioconférence afin de faciliter l'accès à des interviewés de tout le territoire français (y compris les DOM-TOM). La première partie des entretiens fut consacrée aux définitions de l'engagement données par les jeunes, afin, notamment, de préciser quels sont les indicateurs de l'engagement environnemental selon eux. Nous avons posé des questions comme « Selon toi, qu'est-ce qu'une personne engagée ? » ou encore, «À partir de quand as-tu décidé de t'engager dans le domaine de l'environnement? Pourquoi?». La seconde partie des entretiens fut consacrée à des questions permettant de mieux comprendre comment la réception, la diffusion ou la production de contenus en ligne peut être considérée comme une action d'engagement ou non dans le domaine de l'environnement. Ici, nous avons posé des questions comme «Selon toi, est-ce que partager du contenu en faveur de la défense de l'environnement est une action engagée ?» ou encore, «Est-ce que les réseaux sociaux jouent un rôle important dans ton engagement?». Dans les deux parties des entrevues, des questions furent posées aux jeunes concernant leur rapport aux autres et l'affichage de leur engagement sur les réseaux sociaux. Tous les entretiens furent retranscrits à des fins d'analyse.

6 Notre travail d'analyse fut d'abord orienté par une approche inductive, inspirée des étapes de la codification, de la catégorisation et de la mise en relation, telle que présentée dans la théorie ancrée de Glaser et Strauss (1967). Nous avons d'abord procédé à une codification minutieuse des entretiens, afin de relever les unités de sens récurrentes. Par la suite, nous avons regroupé ces unités de sens pour produire les catégories les plus significatives chez les jeunes interviewé.e.s dont nous avons changé les noms pour la restitution des données.

7 Il est apparu que la catégorie du risque était omniprésente dans les discours des jeunes adultes, mais que cette catégorie ne renvoyait pas toujours aux questions de risques environnementaux ou climatiques, mais bien à des formes plus personnelles de « prises de risque » expérimentées dans la rencontre avec l'autre. Le retour vers le contenu des entretiens, cette fois avec l'objectif de préciser cette catégorie de "prise de risque ", nous a permis de voir que les sous-catégories du risque de la révélation, de la désapprobation et de l'accusation traversent les discours des répondant.e.s, et que chacune de ces prises de risque entretient un lien avec le «processus d'expérimentation » évoquée par Pleyers, ce que nous expliciterons dans les sections suivantes.

\section{Le risque de la révélation de l'engagement en faveur de l'environnement}

8 L'usage des réseaux sociaux n'exclut jamais une part d'incertitude et les jeunes de l'enquête nous font part des risques que leurs usages impliquent en ce sens. 
D'emblée, le partage de contenu est présenté bien souvent comme une modalité interactionnelle qui comporte sa part de risque, à la manière de Marine, 23 ans, qui nous explique :

Du moment qu'on partage quelque chose qui est visible, on prend un risque, et qu'on n'est pas protégé, c'est-à-dire que moi je suis en privé, c'est ce que je vous disais, mais si on est en public, dans tous les cas on prend un risque, oui, on prend le risque que quelqu'un vienne nous embêter, que quelqu'un vienne vous insulter, d'être harcelé, voilà, bien sûr. (Marine, 23 ans)

10 Les propos de Marine traduisent la crainte d'un enchaînement de violence qui peut caractériser les échanges en ligne, mais pas seulement. Embêter, insulter, harceler: l'affichage de son engagement, à travers le partage de contenu ou la prise de position en ligne, comporte le risque de devoir se confronter à l'hostilité des autres. Et s'engager « en s'affichant » ne se limite pas à l'exposition de soi sur les réseaux sociaux comme le rappelle Carmina, 25 ans, car « (...) on peut prendre le risque d'être, si y'a des personnes qui sont pas en faveur de l'écologie, on peut avoir des réflexions, mais moi, je me dis pas que je prends un risque " (Carmina, 25 ans). Or, les jeunes de notre enquête sont nombreux à ne pas partager la position de Carmina. Le risque de la confrontation à l'autre, de devoir subir des désagréments sur le mode de la dévalorisation, voire de l'attaque personnelle, amène la plupart d'entre eux à revoir l'intensité de leur affichage à la baisse, comme l'indique Emmanuelle, 21 ans :

Tu prends des risques en partageant n'importe quel type d'information, n'importe lequel littéralement. Moi, mon profil, je l'ai pas mis en public, donc je choisis quelles publications je mets en public et quelles publications je ne mets pas en public. Mais bien sûr, si je publie quelque chose sur l'écologie et que je cherche un travail, et que là un de mes patrons potentiels tombe sur ça et que ça lui plaît pas, bien sûr que je prends un risque, bien sûr, je prends le risque de ne pas être embauchée. (Emmanuelle, 21 ans)

11 La crainte qu'évoque Emmanuelle au sujet de l'opinion de futurs employeurs souligne que l'absence de frontière spatiale et temporelle de la publication en ligne participe d'une représentation du risque qui ne saurait trouver un parfait équivalent dans les espaces physiques. En effet, si l'absence de frontières spatiales à l'interaction en ligne induit la possibilité qu'une multiplicité d'individus devienne des interlocuteurs potentiels au même moment, l'absence de frontières temporelles induit, quant à elle, la possibilité que de nouveaux individus interprètent les contenus dans l'avenir (comme de «futurs » employeurs potentiels). Ainsi, l'espace numérique propice à la diffusion des idées se caractérise par un double régime de contraintes du fait d'une double forme d'incertitude : incertitude de qui "peut nous voir ", incertitude de qui «pourra nous voir », ce qui est largement associé à l'idée d'« audience imaginée » telle que décrite par la chercheure danah boyd (2014) ${ }^{2}$.

12 Ces craintes ne sont d'ailleurs pas que des spéculations évoquées par les répondants. Plusieurs jeunes de notre enquête ont expérimenté eux-mêmes des formes plus ou moins intenses d'hostilité à leur égard.

Ça m'est déjà arrivé. C'est des internautes externes qu'on a que sur la toile, donc même si quelqu'un nous contacte c'est un peu bloqué sur Facebook et on peut ne pas y répondre, y'a plein de gens qui risquent de recevoir un message un peu désobligeant. (Yazmin, 19 ans)

13 La révélation de son engagement en ligne implique ce risque de la confrontation, voire d'insultes, ce qui ne semble d'ailleurs pas rare, car les répondant.e.s nous parlent non seulement de leurs propres expériences, mais aussi de l'expérience de proches qui sont 
aussi engagés en faveur de l'écologie. Il en résulte que l'imaginaire du risque tourne aussi autour de cette possibilité d'être victime de l'hostilité des autres :

Y'a quand même un petit risque de se faire rejeter par certaines personnes, parce que c'est vrai qu'il y a quand même beaucoup de clichés sur les personnes qui sont engagées en écologie, y'a beaucoup de personnes qui disent : “Ah oui, c'est une bobo qui vote à gauche et qui pense qu'à ça, elle ne va pas s'amuser parce qu'elle est dépressive, elle pense que tout le monde va mourir." Alors que ce n'est pas du tout ça, moi je suis une personne très joyeuse au quotidien, je m'implique là-dedans, bon je dirai qu'on m'a déjà fait des remarques qui n'allaient pas aussi loin que ce que je viens de dire, mais qui n'étaient pas très sympas. C'est déjà arrivé à certains de mes amis aussi [...] (Marie-Louise, 18 ans)

Nous comprenons bien que ce risque perçu peut être facilement "surmonté », à la condition d'accepter la possibilité du conflit, d'être bousculé à travers l'expérience du débat, d'accepter l'altérité et parfois la déception, voire des désaccords qui peuvent déboucher sur des conflits, ce qu'évoque bien Mathias, 23 ans, qui accepte cette part d'incertitude qu'implique sa position de jeunes engagés :

\begin{abstract}
Est-ce qu'on prend un risque par rapport à son image notamment? Je pense qu'en fait on prend des risques émotionnels oui, c'est possible, parce qu'on risque de frustrer les amis, on risque de ne pas plaire à des personnes. Et en même temps, finalement, c'est plus un risque, mais au contraire, je pense que c'est bénéfique parce que c'est se mettre soi-même en accord avec ce que l'on pense, de partager des choses qui nous correspondent [...]. Je pense que, finalement, ce risque il est un petit peu flou, c'est juste sur l'apparence, et l'apparence pour moi c'est un petit peu superficiel, c'est plutôt sur le fond qu'il faudrait juger les personnes. (Mathias, 23 ans).
\end{abstract}

Si le fait de surmonter ce risque inhérent à la révélation de son engagement peut être perçu comme la marque d'un engagement réel, nous pouvons émettre l'hypothèse que cette crainte produit sur un certain nombre de jeunes un effet inhibitoire ${ }^{3}$, susceptible de freiner l'expression de leur désir d'engagement, voire d'amener certains à se taire, même à s'en détourner. Car prendre ce risque et le surmonter apparaît comme une condition sine qua non de l'engagement comme "processus créatif d'expérimentation ». Les réactions sont diversifiées et inattendues sur les réseaux sociaux, de la part d'un public aux contours imprécis, d'où l'incertitude et le risque de la réaction "désagréable " et "désobligeante ». C'est donc la capacité à composer avec les arguments et les attaques qui départageraient ici ceux et celles qui acceptent de prendre ce risque de ceux et celles pour qui ce risque pèse encore trop dans la balance des choix à faire pour s'exposer. Nous verrons qu'à ce risque lié à des effets personnellement ressentis par l'individu lors d'une interaction qui n'est pas maîtrisée, s'ajoute le risque, au contraire, de produire un effet qui n'est pas désiré chez l'interlocuteur lors d'une publication en ligne.

\title{
Le risque de la désapprobation de ses opinions en faveur de la protection de l'environnement
}

L'incertitude ne concerne pas seulement la réaction de l'autre à l'égard de soi. Elle concerne aussi l'effet induit chez l'interlocuteur. La plupart des jeunes de notre enquête considèrent sans surprise que la sensibilisation du plus grand nombre aux causes environnementales est cruciale pour l'avancement de leur cause. Le partage de contenu en ligne est un moyen, parmi d'autres, pour s'impliquer dans un mouvement 
de sensibilisation, d'où une grande attention portée aux modalités de diffusion et aux critères justifiant la qualité d'un contenu. À cet égard, la crainte de "soûler " les autres, expression récurrente dans les discours, traduit cette recherche du meilleur équilibre entre le désir de rendre visible son engagement en partageant de l'information d'une part, et la nécessité de ne pas surexposer les interlocuteurs à du contenu "engagé » d'autre part. Comme le mentionne Julien, 24 ans, cet équilibre concerne d'abord la quantité d'information partagée sur ses comptes personnels: "Même moi, y'a des gens des fois qui partagent du contenu, qui partagent tellement de contenu sur quelque chose que j'ai presque envie de me désabonner de ces informations, même si ce sont des informations qui sont intéressantes à la base " (Julien, 24 ans). "Souler les autres » peut certes être contre-productif dans la mesure où le partage « excessif» de contenu engagé peut, selon certains, amener les contacts à se désabonner de leurs comptes et donc à perdre le lien qui permettait jusque-là des échanges au sujet de la cause défendue. C'est du moins ce que nous explique Olga, 23 ans, lorsqu'elle nous parle de cette étiquette qui risque de coller au compte de ceux et de celles qui partagent " beaucoup d'infos sur l'écologie » :

Y a aussi le fait malheureusement des étiquettes qu'on peut nous coller, c'est-à-dire que moi je suis arrivée à une période où maintenant je partage beaucoup d'infos sur l'écologie, entre autres, et du coup ça peut des fois exaspérer certaines personnes justement de voir toujours des infos là-dessus parce qu'elles se sentent hyper éloignées du sujet, et ça les soûle concrètement. (Olga, 23 ans)

Si le risque de mettre à distance des interlocuteurs potentiels se fonde sur la quantité d'informations partagées, ce risque concerne aussi la qualité de celles-ci. Cette fois, les jeunes de l'enquête évoquent la nécessité de vérifier ses sources et donc d'éviter la diffusion d'information imprécise, voire erronée :

Si on donne des informations qui ne sont pas hyper précises, ce qui arrive souvent, donc c'est souvent aussi contestable du coup, et si on partage un article comme ça, même si on a fait gaffe aux sources, même si c'est des choses qui ont été reprises, etc., forcément qu'on prend toujours un risque de pouvoir être contesté, qu'il y ait des gens qui ne soient pas d'accord, qui remettent en question. Donc oui, bien sûr qu'on prend un risque. (Marie, 25 ans)

En prêtant attention au discours de Marie, force est de constater le lien qu'elle établit directement entre la fiabilité de l'information diffusée et la possibilité pour l'interlocuteur de se transformer en détracteur. Tout se passe comme si la fin justifiait les moyens, dans la mesure où ce n'est pas toujours l'intention d'être scientifiquement valide qui compte, mais bien l'intention d'être publiquement validé (Lachance et Przygoda, 2020). En ce sens, Emma, 22 ans, ne cache pas que l'imprécision des informations qu'elle partage est moins importante que l'effet provoqué chez les interlocuteurs :

Même s'il y a de l'information un peu fausse, y'a des fake news qui circulent, à partir du moment où elles servent l'idée de l'écologie, moi j'avoue que ça me dérange pas trop. Mais le risque ce serait que si y'a trop de fake news qui circulent, au bout d'un moment les gens n'y croient plus, ils se disent: “Toute l'écologie c'est des fake news, tout ce qui est en rapport avec l'écologie sur les réseaux on peut pas faire confiance, c'est du greenwashing, ce n'est pas des bonnes sources, les personnes qui fournissent ça sont pas sérieuses." Ça, par contre, ça pourrait être un problème. (Emma, 22 ans)

19 À la quantité excessive d'information et à la validité de leur contenu s'ajoute le problème de la charge émotionnelle que peut provoquer le partage d'un tel contenu. 
Les jeunes de l'enquête ne nous parlent alors plus de l'analyse des interlocuteurs qui peut les amener à douter du discours défendu, mais plutôt des expériences émotionnelles que suscite l'exposition à certains contenus, au point d'engendrer un effet de rejet de la cause écologique. Ainsi, Lisa, 20 ans, attire notre attention sur les effets contreproductifs des images partagées sur les réseaux sociaux: «Les émotions c'est important, mais si y'a trop d'émotions, par exemple avec les vidéos d'abattoirs, les choses comme ça, ou juste dire à la personne "on va tous mourir" ou "à cause de toi y'a des gens à l'autre bout du monde qui souffrent", de toute façon si y'en a trop, la personne risque juste de se renfermer. » (Lisa, 20 ans)

Nadine, 18 ans, abonde aussi dans ce sens, dans la mesure où la gravité de ce qui est partagé peut amener l'interlocuteur à rejeter en bloc la véracité de ce qui transmis : " ça peut vraiment faire paniquer certaines personnes, ou alors certaines personnes se braquent et disent: «Non, ce n'est pas vrai, c'est n'importe quoi. » Difficile donc de prévoir la réaction de l'autre en ligne.

21 À défaut de pouvoir maîtriser les effets provoqués par le partage d'information, il est néanmoins possible pour les jeunes de l'enquête de chercher l'équilibre sur les registres de la quantité en gérant le flux du contenu diffusé, de la qualité en évitant le partage d'informations erronées, et de ne pas oublier les effets possibles sur certaines personnes d'un contenu choquant. Au final, c'est moins le risque de la désinformation dont nous parlent les jeunes de l'enquête que le risque de la désapprobation. Car c'est bien de la validation de la démarche empruntée pour convaincre dont il est ici question. Le double registre de l'expérimentation réapparaît alors dans le contexte du partage de contenu : pour faire valider le contenu partagé, il importe de faire valider aussi la modalité de partage, qui demande précisément de trouver l'équilibre sur les registres de la quantité, de la fiabilité de la source et de l'émotion suscitée. Une fois de plus, à défaut de repère précis, il s'agit d'expérimenter certains usages, de vivre avec l'incertitude, cette fois, au sujet des effets provoqués, et de prendre un risque éminemment relationnel.

\section{Le risque de l'accusation de transgression et de délit}

Le brouillage des frontières temporelles et spatiales de l'échange sur les réseaux sociaux contribue non seulement à l'existence de cette incertitude concernant la réaction suscitée chez soi et chez l'autre, mais aussi à l'avènement du risque d'être vu et repéré par des personnes auxquelles les contenus ne sont pas destinés. Ainsi les répondants de notre enquête nous parlent à plusieurs reprises du fait qu'ils sont, selon eux, «surveillé ». C'est du moins ce qu'évoque Daniel, 23 ans, qui considère que son activité en ligne comporte même le risque d'être "fiché » par un gouvernement qu'il considère éventuellement comme hostile à l'égard des personnes engagées dans le domaine de l'écologie comme lui : «Je pense que oui, on peut prendre des risques étant donné qu'il y a, et on l'a appris récemment, un fichage des militants écologistes qui est fait par le gouvernement et du coup, oui, partager ce genre d'information sur internet peut être un risque à ce niveau-là » (Daniel, 23 ans). Pour Fabrice, 19 ans, ce risque inhérent à la surveillance gouvernementale s'ajoute d'ailleurs à celui concernant de futurs employeurs. En tant que personnes engagées en faveur de l'écologie, différents acteurs peuvent selon lui, considérer ses actes comme étant répréhensibles : 
C'est plutôt des risques de surveillance parce qu'il y a plusieurs aspects, y'a le fait que, je sais plus si c'est le Ministère de l'Intérieur ou les renseignements directement, qui disent que les écolos et les véganes c'est des terroristes du futur et que, du coup, ils vont les ajouter à certaines nomenclatures de fichiers de renseignements. Et aussi, la création d'un fichier sur l'écologie dans le milieu agricole spécifiquement. Donc, il y a des risques vis-à-vis du fichage par les services de renseignements et de surveillance des activités écologiques, etc., et donc de sa vie personnelle. Après, il y a peut-être potentiellement des risques vis-à-vis des employeurs. (Fabrice, 19 ans)

Une fois de plus, si les espaces numériques apparaissent comme des réseaux de prédilection pour toucher le plus grand nombre, et susciter l'intérêt pour la cause défendue, en même temps, l'absence de frontières spatiales et temporelles provoque l'existence de nouveaux risques qui n'impliquent pas toujours le contexte actuel de l'échange ou de la publication, mais bien les contextes à venir dans lesquels le contenu sera réinterprété.

Les jeunes de l'enquête ont donc bien souvent conscience de la permanence de traces laissées par leur activité en ligne. C'est ce que soulignent les témoignages de Lucas, 18 ans, et de Victoria, 25 ans. Pour le premier, les messages qu'il envoie et le contenu qu'il partage n'est pas un problème dans l'immédiat. Mais la récupération de ce qu'il publie et échange maintenant dans un avenir qu'il imagine se transformer est à même, paradoxalement, de troubler la quiétude d'une publication dans le présent. En d'autres termes, le contexte imaginé de l'avenir est réintroduit dans le contexte du présent :

Il y a eu plusieurs cas de comptes Facebook ou même Instagram qui ont été bloqués parce qu'ils publiaient des messages engagés, des messages de désobéissance civile, des appels à la désobéissance civile et du coup, on se demande jusqu'où ça va aller. Actuellement, je pense qu'on ne s'expose pas vraiment à de grosses conséquences, mais peut-être qu'à l'avenir ça se durcira, on sait pas trop. (Lucas, 18 ans)

Le discours est semblable chez Victoria lorsqu'elle évoque que, dans certaines circonstances, les publications en ligne peuvent mettre en danger les militants auxquels elle s'identifie :

Ça dépend de ce qu'on partage, ça dépend vraiment de ce qu'on partage, si par exemple c'est quelque chose qui peut potentiellement être affilié à des groupes soidisant extrêmes pour les forces de l'ordre, oui c'est un risque, en tout cas, ça peut être aggravant en cas de procès ou de garde à vue. Mais pour moi, le risque il est pas énorme, du moment où on n'est pas fiché par les services de police ou que ça soit pas une action offensive envers une entreprise. Enfin, pour moi c'est pas trop risqué, mais ça peut être aggravant dans certaines situations, en cas de procès notamment. (Victoria, 25 ans)

Une fois de plus, les craintes de Lucas et de Victoria posent les deux questions suivantes: «qui peut nous voir?» et «qui pourra nous voir?», rappelant que l'abolition des frontières spatiales et temporelles de l'échange en ligne implique l'expérimentation créative dans le présent de la publication, d'une autre forme de prise de risque. Julien, 24 ans, abonde dans le même sens, lorsqu'il évoque l'éventualité d'un changement de « régime gouvernemental»:

J'ai peur qu'un jour y'ait des dérives qui soient faites en fonction du régime gouvernemental, et qu'il arrive qu'il y ait des représailles contre des personnes qui sont trop engagées on va dire. (Julien, 24 ans)

27 Au risque de la confrontation et de la désapprobation s'ajoute finalement le risque de l'accusation, dans la mesure où des prises de position affichées en ligne peuvent, de l'avis de nombreux jeunes rencontrés, devenir des arguments pour transformer leur 
propos ou leurs actions en délits. $\mathrm{Si}$, en tant que "processus d'expérimentation créatif ", l'engagement comporte toujours une part de prise de risque, le contexte de l'échange en ligne rappelle que le paramètre des traces du contenu échangé ajoute une part d'incertitude, du fait de la possible décontextualisation de ces derniers.

\section{Discussion}

Face aux risques de la confrontation, de la désapprobation et de l'accusation, nous imaginons aisément que les enfants et les adolescents ne sont pas tous égaux; certains vivront éventuellement de telles invalidations de leur affichage avec le sentiment plus ou moins important d'être violenté. Rappelons que dans le cadre de notre enquête, nous avons rencontré des jeunes se déclarant eux-mêmes comme étant engagés dans le domaine de l'environnement. Malgré l'affirmation de cet engagement, voire sa revendication, les freins à l'affichage de ce même engagement sont apparus dans leurs discours. Ainsi pouvons-nous émettre l'hypothèse que certains jeunes ayant une faible estime d'eux-mêmes ne pourraient résister à l'effet inhibitoire de ces prises de risque, dont la valeur symbolique peut certainement varier d'une personne à une autre. En d'autres termes, le risque de la confrontation, de la désapprobation et de l'accusation ne sont probablement pas évaluées avec le même degré d'intensité chez les jeunes, comme le montrent les propos exposés dans cet article : de " petits risques " à " risques significatifs ", le regard de l'autre ne pèse jamais exactement du même poids dans la balance des choix effectués par un.e répondan.et et un.e autre.

Le rôle de la prise de risque dans la formation de l'identité a été vastement documenté (Peretti-Watel, 2002 ; Jeffrey et coll., 2005). Les prises de risque symboliques, comme dans les exemples rapportés dans cet article, fortifient la confiance en soi lorsque le résultat espéré est obtenu, mais elles ont pour effet de l'affaiblir en cas d'échec. Dans un contexte où le « processus d'expérimentation » caractérise l'engagement, les prises de risque sont nombreuses, et les possibilités de renforcer cette confiance en soi le sont aussi. En même temps, les incertitudes qui leur sont inhérentes complexifient le parcours de l'engagement pour des jeunes plus «fragiles", au sens où le risque pris peut-être trop lourd à supporter, les avantages perçus à s'affirmer et à afficher ses convictions apparaissant éventuellement trop lointains et trop abstraits. Cette observation n'est pas anodine, car cela signifie qu'un travail impératif sur l'estime de soi serait nécessaire, incontournable, afin de favoriser, non pas l'adhésion à la cause environnementale, mais plus précisément, la possibilité de l'exprimer chez des jeunes déjà convaincus. Nous comprenons d'emblée que certains groupes de pairs vont favoriser cette expression, tout en faisant courir un autre risque aux adolescents et aux adolescentes concerné.e.s : celui de s'en tenir à l'expression de son engagement dans des groupes relativement refermés sur eux-mêmes, sans possibilité de porter la cause au-delà de ses frontières.

Pour les acteurs du champ de l'éducation relative à l'environnement, cela signifie qu'une attention devrait être portée au fait qu'il existe un écart entre, d'une part, le fait d'adhérer à la cause environnementale et, d'autre part, le fait d'exprimer cette adhésion. Il est alors imaginable que des professionnels au contact de jeunes interprètent parfois leur attitude comme une forme de désintérêt pour la cause environnementale alors que, dans les faits, c'est la difficulté à s'exprimer à ce sujet qui s'impose, précisément afin d'éviter les risques de révélation, de désapprobation et 
d'accusation qui sans doute, n'apparaisse pas seulement sur les interactions en ligne, mais aussi hors ligne. Il serait en ce sens pertinent d'identifier les freins et les leviers à l'expression des convictions dans le contexte des interactions sociales, en sachant que le contexte normatif de ces interactions est susceptible de ralentir, non seulement son expression, mais aussi son développement, ce que notre étude n'a pas permis d'approfondir à ce jour. Plus encore, il serait judicieux de vérifier si le choix d'une certaine partie de la population des jeunes pour un engagement s'exprimant davantage dans la sphère privée ne serait pas en partie lié à une volonté d'éviter les risques de confrontation, de désapprobation et d'accusation que nous avons évoqués dans cet article.

\section{BIBLIOGRAPHIE}

Balleys, C., 2018, » Socialisation adolescente et usages des médias sociaux : la question du genre ». Revue des politiques sociales et familiales, numéro thématique Parcours adolescents : expériences et représentations, 125 , p.33-44.

Beck, U. (1992). Risk society : Towards a new modernity. Sage Publications.

Bell, B. T., Toth, N., Little, L. et Smith, M. A. (2016). Planning to Save the Planet : Using an Online Intervention Based on Implementation Intentions to Change Adolescent Self-Reported EnergySaving Behavior. Environment and Behavior, 48(8), 1049-1072.

Bennett L., 2013, " "If we stick together we can do anything" : Lady Gaga fandom, philanthropy and activism through social media ». Celebrity Studies, 5(1-2), 138-152

Boulianne, S. (2009). Does Internet Use Affect Engagement? A Meta-Analysis of Research. Political Communication, 26(2), 193-211.

Boulianne, S. (2011). Stimulating or Reinforcing Political Interest : Using Panel Data to Examine Reciprocal Effects Between News Media and Political Interest. Political Communication, 28(2), 147-162.

Boulianne, S. (2015). Social media use and participation : A meta-analysis of current research. Information, Communication et Society, 18(5), 524-538.

Boyd, D. (2014). It's complicated : The social lives of networked teens (1. Aufl). New Haven, Connecticut, États-Unis : Yale University Press.

Chawla, L. (1998). Significant Life Experiences Revisited : A Review of Research on Sources of Environmental Sensitivity. The Journal of Environmental Education, 29(3), 11-21.

Chawla, L. et Cushing, D. F. (2007). Education for strategic environmental behavior. Environmental Education Research, 13(4), 437-452.

Conroy, M., Feezell, J. T. et Guerrero, M. (2012). Facebook and political engagement : A study of online political group membership and offline political engagement. Computers in Human Behavior, 28(5), 1535-1546. 
Dahlgren P. (2012), Web et participation politique : quelles promesses et quels pièges ?, Questions de communication, no 21 , p. 13-24

Fuchs C., 2014, « Twitter and democracy : A new public sphere?». Dans C. Fuchs, Social Media. A critical introduction, (Chapitre 8). Los Angeles, London : Sage.

Glaser, B. G. et Strauss, A. L. (1967). The discovery of grounded theory : Strategies for qualitative research (4. paperback printing). Aldine.

Gravel, H. et Pruneau, D. (2004). Une étude de la réceptivité à l'environnement chez les adolescents. Revue de l'Université de Moncton, 35(1), 165-187.

Ion, J. (1997). La fin des militants ? Ivry-sur-Seine : Éditions de l'Atelier/Éditions Ouvrières. Jeffrey, D., Le Breton, D., et Lévy, J. J. (Dir.). (2005). Jeunesse à risque, rite et passage. Québec : Les Presses de l'Université Laval.

Lachance, J. (2018). L'adolescence hypermoderne à l'heure de l'accélération sociale. Dans @ la recherche du temps (p. 295-307). ERES ; Cairn.info.

Lachance, J. et Przygoda, M. (2020). Scientific Data in the Ecological Commitment of Young People, In the Digital Age. Interdisciplinary Journal of Environmental and Science Education, 17(1), e2229. Consulté sur www.ijese.com/download/scientific-data-in-the-ecological-commitment-ofyoung-peoplein-the-digital-age-9156.pdf

Livingstone, S. et Helsper, E. (2007). Gradations in digital inclusion : Children, young people and the digital divide. New Media et Society, 9(4), 671-696.

Musick, M. A. et Wilson, J. (2008). Volunteers : A social profile. Bloomington, Indiana, États-Unis : Indiana University Press.

Parot, F. et Richelle, M. (2013). I. Introduction. Dans Introduction à la psychologie (p. 7-15). Paris : Presses Universitaires de France. Consulté sur www.cairn.info/introduction-a-lapsychologie--9782130619802-p-7.htm

Peretti-Watel, P. (2002). Les « conduites à risque » des jeunes : Défi, myopie, ou déni ? Agora débats/jeunesses, 16-33. Consulté sur www.persee.fr/doc/agora_1268-5666_2002_num_27_1_1994

Pizzorno, A. (2009). Rationalité et reconnaissance. Dans C. Lazzeri et A. Caillé (Dir), La reconnaissance aujourd'hui (p. 229-271). Paris : CNRS Éditions.

Pleyers, G. (2010). Alter-globalization : Becoming actors in the global age. Cambridge : Polity Press. Pleyers, G. (2016). Engagement et relation à soi chez les jeunes alteractivistes. Agora débats/ jeunesses, 72(1), 107-122. Consulté sur www.cairn.info/revue-agora-debats-jeunesses-2016-1page-107.htm

Valenzuela, S., Arriagada, A. et Scherman, A. (2012). The Social Media Basis of Youth Protest Behavior : The Case of Chile. Journal of Communication, 62(2), 299-314.

Vitak, J., Zube, P., Smock, A., Carr, C. T., Ellison, N. et Lampe, C. (2011). It's Complicated : Facebook Users' Political Participation in the 2008 Election. Cyberpsychology, Behavior, and Social Networking, 14(3), 107-114.

\section{NOTES}

1. Voir : https://tree.univ-pau.fr/fr/activites-scientifiques/contrats-de-recherche/contratsaxe-1-regulations/ecotic.html 
2. Danah boyd insiste dans ses travaux sur le fait que sur les réseaux sociaux numériques, l'individu qui publie du contenu peut avoir le sentiment d'avoir été vu par ses contacts, sans jamais en être certain, ce qui laisse place à une certaine interprétation quant au silence et à l'absentions de ceux et de celles qui ne commentent pas les publications en ligne.

3. Dans le cadre du projet ECOCOV, nous réalisons actuellement une enquête quantitative à l'échelle nationale qui nous permettra notamment de vérifier la transférabilité de nos observations dans les entretiens réalisés.

\section{RÉSUMÉS}

À partir de l'analyse qualitative du contenu de 32 entretiens réalisés auprès de jeunes se déclarant engagés dans le domaine de l'écologie en France, cet article propose d'analyser de manière inductive les risques perçus par ces derniers lors de l'affichage de leurs convictions sur les questions environnementales en ligne. Comme le souligne le sociologue Geoffroy Pleyers, si l'engagement comporte toujours une part d'expérimentation dans le contexte contemporain, alors la rencontre avec l'autre peut apparaître comme un moment d'incertitude et de prises de risque. Ainsi les jeunes de l'enquête nous font part du risque de la confrontation, de la désapprobation et même de l'accusation dont ils sont parfois victimes. Notre analyse nous amène alors à interroger en quoi les contextes interactionnels peuvent constituer des freins (ou des leviers) à l'engagement des jeunes dans le domaine de l'éducation relative à l'environnement.

Based on the qualitative analysis of the content of 32 interviews carried out with young people declaring themselves engaged in the field of ecology in France, this article proposes to inductively analyze the risks perceived by the latter when posting their beliefs on environmental issues online. As sociologist Geoffroy Pleyers points out, if engagement always involves an element of experimentation in the contemporary context, then meeting with the other can appear as a moment of uncertainty and risk-taking. Thus the young people in the survey share with us the risk of confrontation, disapproval and even accusation of which they are sometimes victims. Our analysis then leads us to question how interactional contexts can act as brakes (or levers) on young people's engagement in the field of environmental education.

\section{INDEX}

Mots-clés : adolescent, engagement, interactions, TIC, reconnaissance

Keywords : adolescent, engagement, interactions, ICT, recognition

\section{AUTEUR}

\section{JOCELYN LACHANCE}

Jocelyn Lachance est maître de conférence HDR en sociologie à l'Université de Pau et des Pays de l'Adour et membre du laboratoire TREE (TRansitions Energétiques et Environnementales). Ses recherches portent sur le devenir adulte dans le contexte contemporain. Il est notamment l'auteur de L'adolescence hypermoderne (2011), La famille connectée (2011) et Les tentatives de 
reconnaissance (2021), le co-directeur d'ouvrages collectifs dont Penser l'adolescence (PUF, 2016) et le co-directeur de la collection Adologiques (Presse de l'Université Laval). 